\title{
SUBGLOTTIC STENOSIS COMPLICATING WEGENER'S GRANULOMATOSIS: SURGICAL REPAIR AS A VIABLE TREATMENT OPTION
}

M. S. Herridge, MD, FRCP $(C)^{\mathrm{a}}$

F. G. Pearson, MD, FRCS $(\mathrm{C})^{\mathrm{b}}$

G. P. Downey, MD, FRCP(C), FCCP $^{\mathrm{a}}$

\begin{abstract}
Wegener's granulomatosis frequently involves the subglottis and trachea, often leading to compromise of the upper airway. Moreover, the stenotic segments may persist or progress despite control of the disease elsewhere in the body. In this report, we describe the cases of five patients with Wegener's granulomatosis who, in addition to nasal, sinus, pulmonary and renal involvement, had symptomatic subglottic or tracheal stenosis. Biopsy specimens from involved sites in the subglottis and trachea were often not diagnostic, and the diagnosis was later confirmed by a positive antineutrophil cytoplasm antibody titer. All patients had clinical remission on standard therapeutic regimens with prednisone and cyclophosphamide but continued to have symptoms of extrathoracic airway obstruction. Three of the five patients underwent primary thyrotracheal anastomosis while their disease was in clinical remission, without postoperative compromise of anastomotic integrity or wound healing despite concurrent use of prednisone and cyclophosphamide. There has been no evidence of local disease recurrence during follow-up periods ranging from 3 months to 14 years. We conclude that surgical intervention is a viable treatment option for patients who have symptomatic stenotic segments of the subglottis and trachea as a result of Wegener's granulomatosis in clinical remission. (J Thorac Cardiovasc Surg 1996;111:961-6)
\end{abstract}

$F^{\mathrm{ni}}$ riedrich Wegener first described what he called "rhinogenic granulomatosis" as a unique clinical entity in $1936 .^{1}$ By 1954 , formalized diagnostic criteria for Wegener's granulomatosis (WG) were established; these included involvement of the respiratory tract with necrotizing granulomatous inflammation coincident with focal necrotizing glomerulonephritis and varying degrees of generalized vasculitis. ${ }^{2}$ Subsequently, the concept of limited WG became well accepted as different authors documented its characteristic morphologic and histopathologic characteristics in isolated anatomic sites. $^{3-7}$ To date, a number of case reports and series have documented WG involving the subglottis and

From the Departments of Medicine, Division of Respiratory Diseases, ${ }^{a}$ and Surgery, Division of Thoracic Surgery, ${ }^{b}$ University of Toronto, and The Toronto Hospital, Toronto General Division, Toronto, Ontario, Canada.

Received for publication Feb. 23, 1995.

Accepted for publication June 14, 1995.

Address for reprints: G. Downey, MD, FRCP(C), El0-212, The Toronto Hospital, General Division, 200 Elizabeth St., Toronto, Ontario M5G 2C4, Canada.

Copyright (C) 1996 by Mosby-Year Book, Inc.

$0022-5223 / 96 \$ 5.00+0 \quad \mathbf{1 2 / 1 / 6 7 0 4 1}$ proximal trachea. ${ }^{8-10}$ This is not a rare manifestation of WG; in a longitudinal study of 158 patients with WG, tracheal stenosis was observed to develop sometime during the course of the disease in $16 \% .^{11}$ In general, this limited form of disease responds to treatment with corticosteroids and cytotoxic agents. ${ }^{12,13}$ Recently, however, we encountered several patients in whom symptomatic upper airway obstruction persisted or progressed despite control of the disease elsewhere.

The feasibility and outcome of reconstructive surgery in these patients have not been well documented. There has been a pervasive pessimism in the literature regarding the role of surgery in the treatment of WG of the subglottis and trachea. McDonald and colleagues ${ }^{12}$ stated that reconstructive surgery was not viable in patients with WG because they believed that concurrent use of immunosuppressive agents would interfere with adequate wound healing. Additionally, they believed that these interventions were impractical because of the possibility that local disease reactivation might lead to disruption of the anastomotic site. These reservations were reiterated by Hoare and colleagues, ${ }^{14}$ who used laser vaporization to clear indolent sub- 
glottic stenoses but considered that extensive laryngeal reconstructive procedures would invariably fail because of disease recurrence.

In this report, we describe a series of five cases of subglottic stenosis related to WG. Three of the patients in this series have undergone successful resection and reconstruction of their tracheal stenosis during disease remission on a regimen of immunosuppressive therapy. We propose that surgical intervention is a viable option in selected patients whose disease has been initially controlled with medical therapy.

\section{Case reports}

CASE 1. A 36-year-old man was seen in 1984 with sinusitis, episcleritis, pulmonary infiltrates, polyarthritis, mononeuritis multiplex, and significant constitutional symptoms. WG was diagnosed by means of an open-lung biopsy. Treatment with pulsed methylprednisolone and cyclophosphamide was initiated, with good clinical response. Clinical remission was eventually achieved in 1992, and steroid therapy was tapered. In July 1993, the patient had increasing hoarseness, dyspnea, decline in exercise tolerance, and stridor. Results of tests for antineutrophil cytoplasm antibody (ANCA) were positive, and gallium scan showed increased uptake in both sinuses, the upper airway, and lung fields. Spirometry showed an extrathoracic airway obstruction, and computed tomographic scan of the trachea confirmed a $3 \mathrm{~cm}$ subglottic stenosis with an intact distal tracheobronchial tree. Therapy was initiated with methotrexate because of concerns about persistently low leukocyte count after cyclophosphamide therapy. The patient continued to have symptoms, and rigid bronchoscopy with dilation was undertaken in March 1994. At the time of this procedure, active upper airway disease was noted, as evidenced by septal perforation and nasopharyngeal crusts. Biopsy samples from the subglottic and nasal areas showed nonspecific chronic inflammation. Tests for circulating ANCA (C-ANCA) and antiproteinase 3 remained positive. Therapy with prednisone and cyclophosphamide was initiated to achieve clinical remission before attempted tracheal reconstruction. This patient continues to have clinical remission and is awaiting clinical reevaluation for consideration for surgical repair.

CASE 2. A 48-year-old woman was initially seen in 1979 with sinusitis, pulmonary infiltrates, and renal failure. WG was confirmed by means of an open-lung biopsy. The patient subsequently had dyspnea and stridor, and a $2 \mathrm{~cm}$ tracheal stenosis $1 \mathrm{~cm}$ below the vocal cords was documented by computed tomographic scan. The patient underwent rigid bronchoscopy with dilation and biopsy. Pathologic examination showed nonspecific granulation tissue with focal squamous metaplasia. Therapy with prednisone and cyclophosphamide was initiated, and the disease remitted. In July 1980, the patient underwent elective resection of the stenosis with thyrotracheal anastomosis. A Jackson tracheostomy was placed below the region of the anastomosis because of its close proximity to the cords. Before operation, the patient was receiving 20 mg prednisone every 2 days and $125 \mathrm{mg}$ cyclophosphamide daily. The patient received perioperative steroid coverage, and after operation prednisone was restarted at a dosage of $10 \mathrm{mg}$ daily. Cyclophosphamide was restarted on the third postoperative day at a dose of $125 \mathrm{mg}$ daily. Four weeks after the procedure, the Jackson tube was removed and the tracheostomy stoma was allowed to close. The patient remained on a regimen of prednisone and cyclophosphamide for an additional 2 years, at which time her disease remitted and the medications were tapered. During the past 14 years, since the time of operation, there has been no recurrence of WG, and the anastomosis has remained intact without recurrent stenosis.

CASE 3. A 29-year-old man was seen in 1983 with subglottic stenosis. The patient first came to medical attention at the age of 10 years with arthralgia, abdominal pain, vasculitic skin rash, and renal failure. Findings in several renal biopsy samples were not specific enough for diagnosis. A tentative diagnosis of Henoch-Schönlein purpura was made at that time. At the age of 17 years, the patient required regular dialysis, and he underwent an initial unsuccessful cadaveric renal transplant followed by a successful renal transplant from a living relative. He received immunosuppression with prednisone and cyclosporine A. In 1983, marked stridor developed, with severe nasal and laryngeal inflammation. A biopsy sample of inflamed oral mucosa demonstrated granulomatous inflammation and vasculitic changes consistent with WG. Cyclophosphamide induced clinical remission, and cyclosporine A was discontinued. Prednisone was added to his regimen several years later, when the cyclophosphamide dose was tapered. In June 1993, symptoms of stridor on vigorous exertion developed, and at another medical center the patient underwent elective resection of a $2.5 \mathrm{~cm}$ subglottic stenosis with primary anastomosis. At the time of operation, he was taking $20 \mathrm{mg}$ prednisone every 2 days and $50 \mathrm{mg}$ cyclophosphamide daily. A violent vomiting episode 5 days after operation resulted in incomplete dehiscence of the primary anastomosis and subcutaneous emphysema. The latter resolved after insertion of a Penrose drain. In August 1993, the patient had recurrent dyspnea and stridor necessitating tracheostomy. Computed tomographic scan demonstrated a $2 \mathrm{~cm}$ subglottic stenosis close to the anastomotic site. Bronchoscopy showed evidence of active WG at the carina and main stem bronchi. Therapy with high-dose prednisone and cyclophosphamide was initiated. Three months later, the patient's disease remitted and he underwent stenotic resection with primary thyrotracheal anastomosis. A Montgomery T-tube was left in place as a stent. At the time of operation, the patient was receiving $15 \mathrm{mg}$ prednisone every 2 days. He received perioperative steroid coverage, but prednisone was withheld until postoperative day 10. Cyclophosphamide was reinstituted on third postoperative day 3. Currently, this patient's disease remains in remission and he continues on his immunosuppressive regimen. His anastomosis remains intact 18 months after operation, without evidence of recurrent stenosis.

CASE 4. A 39-year-old woman was seen in 1991 with frontal headache, earache, swollen gums, and constitu- 
tional symptoms. A transnasal biopsy sample showed granulomatous inflammation consistent with WG. The patient was started on a regimen of prednisone, cyclophosphamide, and trimethoprim-sulfamethoxasole, with good clinical response. Nine months later, she had exertional dyspnea, decline in exercise tolerance, and occasional inspiratory stridor. She was admitted to our institution, and flexible bronchoscopy showed an inflammatory web with tracheal narrowing involving $2 \mathrm{~cm}$ of subglottic trachea. There was also evidence of distal disease of the main stem bronchi and lobar orifices. Results of C-ANCA testing were positive. Rigid bronchoscopy with dilation of the stricture was performed. Biopsy samples from the affected sites showed nonspecific chronic inflammation. Cyclophosphamide was discontinued because of bone marrow suppression; this therapy was replaced with methotrexate and azathioprine. Methotrexate was subsequently discontinued, and the patient's disease has remained in remission. There has been no recurrence of upper airway obstruction in 22 months of follow-up.

CASE 5. A 29 -year-old woman was initially seen in 1990 with acute renal failure. Subsequent renal biopsy confirmed WG. Results of C-ANCA testing were positive at that time. The patient was maintained on a regimen of prednisone and cyclophosphamide after acute treatment with plasmapheresis and remained well until April 1990, when she had acute upper airway obstruction. Bronchoscopy demonstrated subglottic stenosis with a $4 \mathrm{~mm}$ residual orifice. Concurrent nasal involvement was present at that time. Despite three laser resections and T-tube placement, the patient continued to require a tracheostomy because of residual stenosis. In September 1994, she was referred to our center for further management. Another bronchoscopy and laryngoscopy demonstrated an anterior subglottic web with normal posterior glottis and a circumferential tracheal stenosis $1.2 \mathrm{~cm}$ in length beginning $1.8 \mathrm{~cm}$ below the vocal cords. These findings were confirmed by computed tomographic scan of the trachea. In anticipation of operation, the patient's maintenance dose of $50 \mathrm{mg}$ prednisone was gradually tapered to $5 \mathrm{mg}$ on alternating days. She continued to take $75 \mathrm{mg}$ cyclophosphamide daily. In November 1994, she underwent resection of the first, second, and third tracheal rings, partial cricoid resection, and laryngofissure and laryngoplasty for posterior stenosis. A T-tube was introduced at that time. Pathologic examination of the resected segment demonstrated nonspecific chronic inflammation. The patient received perioperative steroid coverage and resumed taking 5 mg prednisone on alternating days on the second postoperative day. Cyclophosphamide at a dosage of $75 \mathrm{mg}$ per day was continued until the day of operation and was resumed on the second postoperative day. The patient's disease remains in clinical remission and she has an intact primary anastomosis 6 months after operation.

\section{Operative technique}

In all five cases reviewed in this report, the proximal margin of the pathologic area involved the soft tissues of the subglottic airway in circumferential fashion. Anatomically, the subglottic airway extends from the inferior border of the vocal folds to the lower border of the cricoid cartilage. The distal extent of the pathologic process extends into the upper cervical trachea for a variable distance. The longest stenosed segment observed in these five cases was $3 \mathrm{~cm}$.

In the three patients whose disease was managed by resection and reconstruction with primary anastomosis, a circumferential segment of the upper airway was removed. This segment included a part of the cricoid cartilage. This technique was originally described by one of us in $1975,{ }^{15}$ and a detailed description of this technique was recently published by Maddaus and Pearson. ${ }^{16}$ Figs. 1 through 3 illustrate diagrammatically the technique of resection.

\section{Discussion}

Stenosis of the subglottis and proximal trachea is an important complication of WG. Although only $2 \%$ of patients are initially seen with this manifestation, $16 \%$ of patients have this complication at some time during the course of their disease. ${ }^{11}$

A series of 17 patients with subglottic or tracheal involvement was studied at the Mayo Clinic between 1964 and 1982. ${ }^{12}$ In all affected patients, there was evidence of another site of disease involvement at presentation, and all had evidence of concurrent nasal involvement. Fourteen of these patients had symptoms of hoarseness and progressive stridor. Tissue histologic examination often yielded only nondiagnostic changes of chronic inflammation. These authors reported a uniformly excellent response to conventional therapy with cyclophosphamide and corticosteroids.

Our patients shared many characteristics with this group. All had nasal involvement at presentation with subglottic stenosis and sparing of the distal trachea. Other organ systems were less frequently involved (Table I). Biopsy specimens from areas of active tracheal involvement frequently did not show diagnostic changes, showing evidence only of nonspecific inflammation. In each of our cases, clinical remission was achieved with a standard protocol of cyclophosphamide and prednisone. ${ }^{11}$ The patients in our series, however, had persistent and symptomatic tracheal stenosis despite previous successful medical therapy, laser treatment, and dilation. One patient continued to require a tracheostomy despite all of these different treatment modalities.

The diagnosis of subglottic stenosis as a result of WG is often difficult. Evidence of vasculitic changes, giant cells, and granulomata are frequently absent in specimens obtained from the upper respiratory 


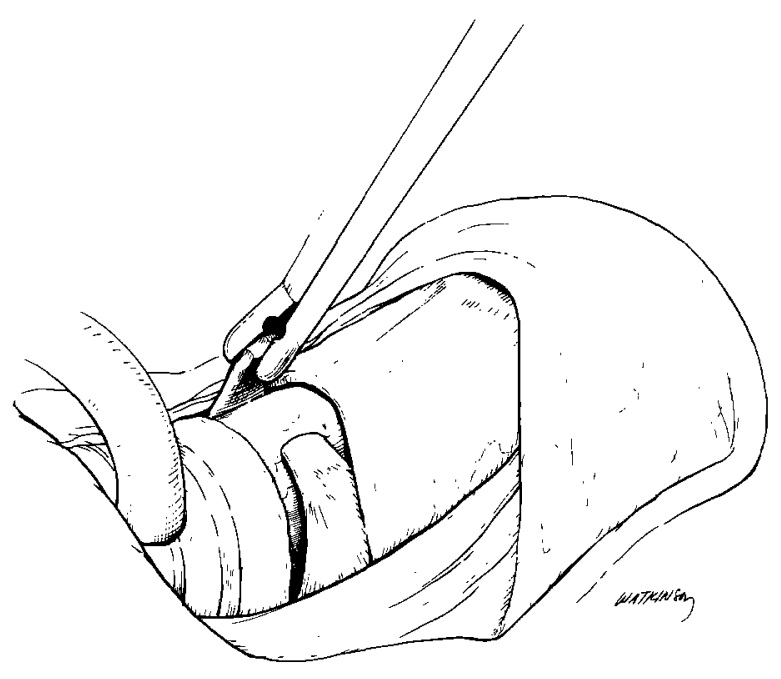

Fig. 1. Diagram illustrating beginning of resection of anterior cricoid arch. The arch has been freed subperichondrally, and illustration shows cartilage being removed with a rongeur.

tract. ${ }^{13}$ Other causes of stenosis, including carcinoma, tuberculosis, syphilis, and lymphoma, need to be excluded because their optimal treatments differ considerably, and any delay contributes to increased morbidity and mortality. Several authors have described the usefulness of ANCA titers in establishing the diagnosis of WG in patients with tracheal stenosis and nonspecific changes of chronic inflammation found in a biopsy specimen. ${ }^{14,17,18}$ Hoare and colleagues ${ }^{14}$ observed that all four patients with WG and subglottic stenosis in their series had a detectable ANCA titer. They advocated the routine use of ANCA assay for patients with subglottic stenosis of uncertain origin. ${ }^{14}$ In three of our five cases, C-ANCA assay was used to establish the presence of active WG (Table I). In the other two cases, a tissue diagnosis was made and ANCA testing was not carried out. Our observations further underscore the clinical usefulness of ANCA testing in the setting of recurrent subglottic stenosis with nonspecific histologic features.

In our series of five patients, three successfully underwent tracheal reconstruction while receiving immunosuppressive therapy. It is important to note that none of these patients had significant postoperative morbidity and none has died. Patients 2,3 , and 5 all underwent primary thyrotracheal anastomoses while their disease was in remission on a regimen of prednisone and cyclophosphamide. All received perioperative steroid coverage and cyclo-
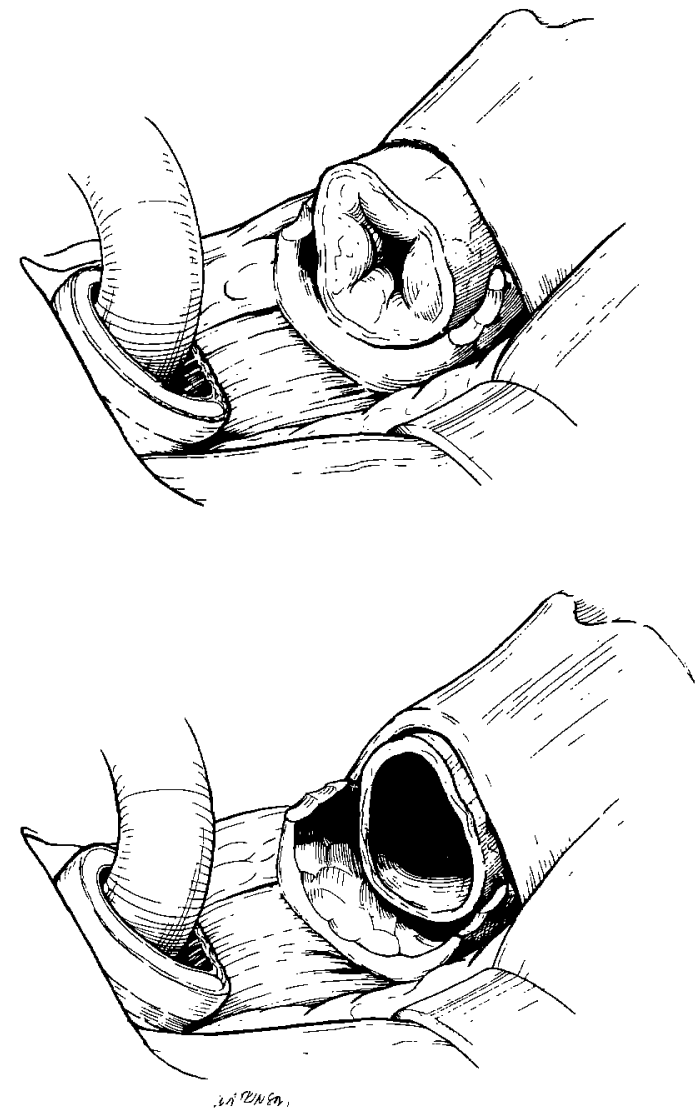

Fig. 2. Diagram illustrating appearance after division of the cervical trachea distal to pathologic area. Distal tracheal stump is intubated, and pathologic area is seen lying within the cricoid cartilage. Entire anterior arch has been removed, leaving only a posterior shell of cricoid cartilage (top). The upper margin of the stenosed subglottic segment is divided at the level of the inferior border of thyroid cartilage (bottom).

phosphamide was restarted 2 to 3 days after operation. It is noteworthy that every effort was made to minimize the dose of corticosteroids given in the perioperative period.

In summary, we have defined a group of patients who had persistent tracheal stenosis despite previous therapeutic interventions. Patients in this subgroup should be offered resection and primary reanastomosis as a definitive reconstructive procedure to obviate the need for tracheostomy and improve functional status and quality of life. Our patients had uniformly excellent clinical outcomes, and thus far there has been no evidence of disease recurrence at the anastomotic site or in any other organ systems. We conclude that primary thyrotracheal anas- 

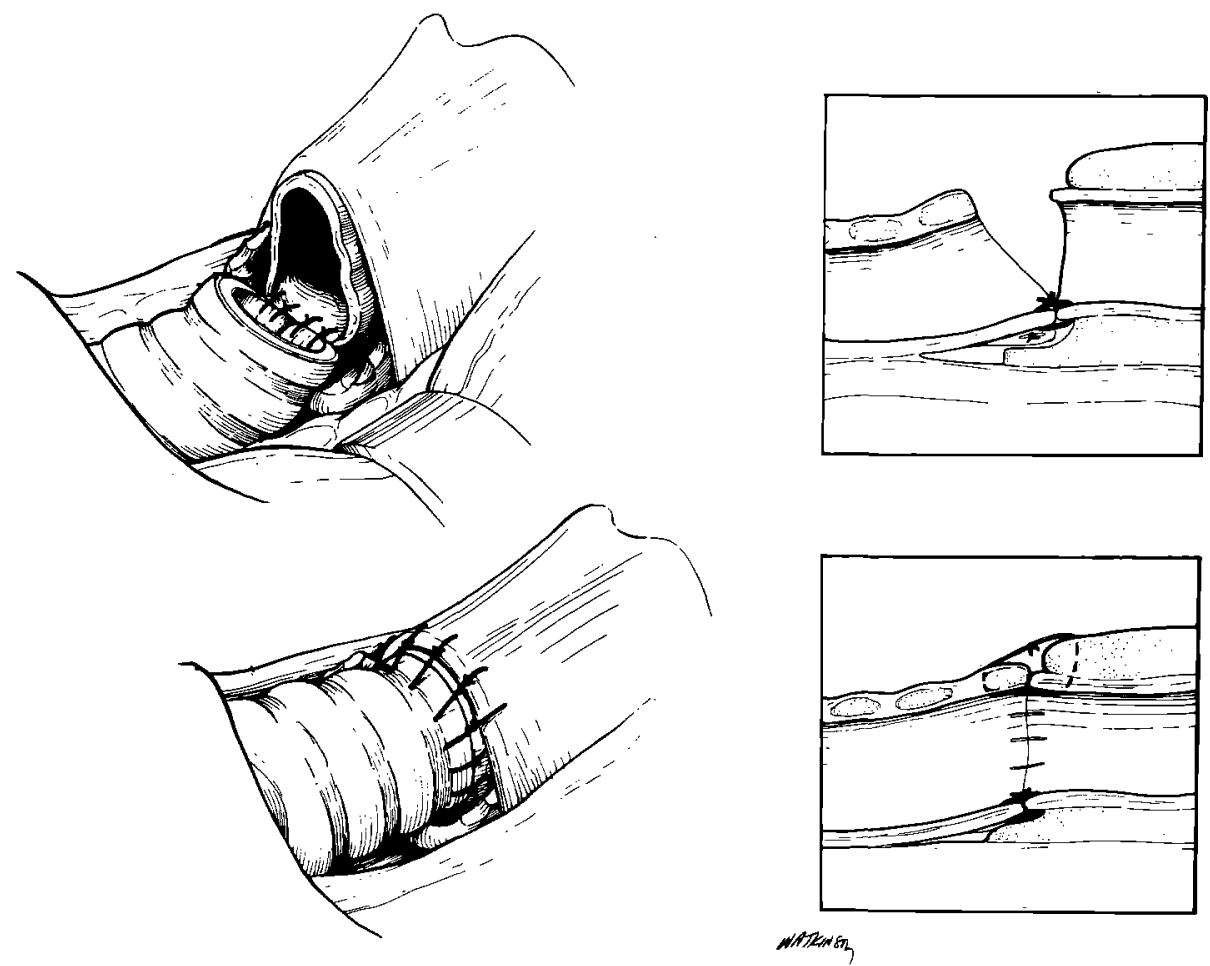

Fig. 3. A primary anastomosis is created between distal trachea and subglottic airway at the level of the thyroid cartilage, identified in the left panel of the diagram. The technique of interrupted suture is shown in the right panel. As shown, the anastomosis lies at the level of the lower border of the thyroid cartilage, and the posterior part of the anastomosis lies anterior to the remaining posterior cricoid plate.

Table I. Summary of patient characteristics

\begin{tabular}{|c|c|c|c|c|c|c|c|}
\hline $\begin{array}{l}\text { Patient } \\
\text { no. }\end{array}$ & $\begin{array}{c}\text { Age at } \\
\text { presentation } \\
(y r)\end{array}$ & $\operatorname{Sex}$ & $\begin{array}{c}\text { Site of } \\
\text { stenosis } \\
\text { and length } \\
\text { of segment }\end{array}$ & $\begin{array}{l}\text { Years after } \\
\text { operation }\end{array}$ & Pathology & $\begin{array}{l}\text { Other organ } \\
\text { involvement }\end{array}$ & $\begin{array}{l}A N C A \\
\text { titer }\end{array}$ \\
\hline 1 & 36 & $\mathrm{~F}$ & Subglottic, $3 \mathrm{~cm}$ & NA & Nonspecific inflammation & Sinuses, nasal, lung & + \\
\hline 2 & 33 & $\mathrm{~F}$ & Subglottic, $2 \mathrm{~cm}$ & 14 & Nonspecific inflammation & Sinuses, nasal, lung, renal & Not done \\
\hline 3 & 29 & M & Subglottic, $2 \mathrm{~cm}$ & 1 & $\begin{array}{l}\text { Granulomatous } \\
\text { inflammation }\end{array}$ & Oral, nasal & Not done \\
\hline 4 & 39 & F & Subglottic, $2 \mathrm{~cm}$ & NA & $\begin{array}{l}\text { Granulomatous } \\
\text { inflammation }\end{array}$ & Oral, nasal & + \\
\hline 5 & 21 & $\mathrm{~F}$ & Subglottic, $1.2 \mathrm{~cm}$ & 0.17 & Nonspecific inflammation & Nasal, renal & + \\
\hline
\end{tabular}

tomosis is a viable treatment option in patients with WG and symptomatic subglottic stenosis whose disease is in clinical remission on a regimen of appropriate immunosuppressive therapy for WG.

\section{REFERENCES}

1. Wegener F. Über generalisierte, septische Gefasserkrangungen. Verh Dtsch Pathol Ges 1936;29:202-9.

2. Godman GC, Churg J. Wegener's granulomatosis: pathology and review of the literature. Arch Pathol 1954;58:533-53.

3. Cassan SM, Coles DT, Harrison EG, et al. The concept of limited forms of Wegener's granulomatosis. Am J Med 1970;49:366-79.

4. Carrington $\mathrm{CB}$, Liebow AA. Limited forms of angiitis and granulomatosis of Wegener's type. Am J Med 1966;41:497527.

5. De Remee RA, McDonald TJ, Harrison EJ, et al. Wegener's granulomatosis, anatomic correlates, a proposed classification. Mayo Clin Proc 1976;51:777-81.

6. Anderson CL, Stavrides A. Rapidly progressive renal failure as the primary manifestation of Wegener's granulomatosis. Am J Med Sci 1978;275:109-12.

7. Baker SB, Robinson DR. Unusual renal manifestations of 
Wegener's granulomatosis: report of two cases. Am J Med 1978;64:883-9.

8. Arkins J, Eisman S. Wegener's granulomatosis. Ann Otol Rhinol Laryngol 1959;68:524-47.

9. Talerman A, Wright D. Laryngeal obstruction due to Wegener's granulomatosis. Arch Otolaryngol 1972;96:376-9.

10. Illum $\mathbf{P}$, Thorling $\mathbf{K}$. Wegener granulomatosis: long term results of treatment. Ann Otol Rhinol Laryngol 1981;90:231-5.

11. Hoffman GS, Ken GS, Leavitt RY, et al. Wegener's granulomatosis: an analysis of 158 patients. Ann Intern Med 1992;116:488-98.

12. McDonald TJ, Neel HB, DeRemee RA. Wegener's granulomatosis of the subglottis and the upper portion of the trachea. Ann Otol Rhinol Laryngol 1982;91:588-92.

13. Arauz JC, Fonseca R. Wegener's granulomatosis appearing initially in the trachea. Ann Otol Rhinol Laryngol 1982;91: 593-4.
14. Hoare TJ, Jayne D, Rhys Evans P, Croft CB, Howard DJ. Wegener's granulomatosis, subglottic stenosis and antineutrophil cytoplasm antibodies. J Laryngol Otol 1989;403:118791.

15. Pearson FG, Cooper JD, Nelems JM, et al. Primary tracheal anastomosis after resection of the cricoid cartilage with preservation of recurrent laryngeal nerves. J Thorac Cardiovasc Surg 1975;70:806-16.

16. Maddaus M, Pearson FG. Subglottic resection. In: Pearson FG, et al. Thoracic surgery. New York: Churchill Livingstone, 1995:chap 16, 321-33.

17. Hoare TJ, Rhys Evans PH. Anti-neutrophil cytoplasmic antibody assay in diagnosis of recurrent subglottic stenosis [Letter]. Lancet 1988;2:1360.

18. Gans RO, Goldschmeding R, Donker AJ, et al. Neutrophil cytoplasmic autoantibodies and Wegener's granulomatosis. Lancet 1989;1:269-70.

\section{ON THE MOVE?}

Send us your new address at least six weeks ahead

Don't miss a single issue of the journal! To ensure prompt service when you change your address, please photocopy and complete the form below.

Please send your change of address notification at least six weeks before your move to ensure continued service. We regret we cannot guarantee replacement of issues missed due to late notification.

\section{JOURNAL TITLE:}

Fill in the title of the journal here.

\section{OLD ADDRESS:}

Affix the address label from a recent issue of the journal here.

\section{NEW ADDRESS:}

Clearly print your new address here.

Name

Address

City/State/ZIP
COPY AND MAIL THIS FORM TO:

Journal Subscription Services

Mosby-Year Book, Inc.

11830 Westline Industrial Dr.

St. Louis, MO 63146-3318
OR FAX TO:

314-432-1158

N/ Mosby
OR PHONE:

1-800-453-4351

Outside the U.S., call

314-453-4351 